\title{
6. DER DICTIONARIUS TRIUM LINGUARUM VON 1513
}

Der Dictionarius: trium linguaru $(m)$. latine. Teutonice: Boemice potiora vocabula continens: peregrinantibus apprime vtilis wurde, wie die Schlußschrift verrät 1513 Vienne Pannon( $n$ ) ie in edibus Hieronymi Vietoris: \& Joannis Singrenii gedruckt ${ }^{183}$. Er hat Quartformat und enthält auf 17 Seiten den Nomenklator aus dem Trialogus, Seite 18 ist - im Gegensatz zur abschließenden Wortliste im Trialogus - ein Numerus. Das Buch ist, wie schon das peregrinantibus des Titels sagt, für den Handgebrauch von Reisenden gedacht, die Druckfläche ist mit zweimal drei Kolumnen pro Seite nicht ästhetisch sondern ökonomisch genützt und verzichtet auf jedweden repräsentativen Gestus, vgl. dazu Abb. 43. Der einzige Schmuck ist ein Holzschnitt auf der Versoseite des letzten Blattes mit dem großen Druckerzeichen des Typographen Hieronymus Viëtor, das mittig den polnischen Adler zeigt, wohl als Ausdruck besonderer landesherrlicher Gunst ${ }^{184}$. In den Ecken darunter sind links das Wappen der Stadt Krakau, rechts das seiner Universität. Links oben sind - wohl seitenverkehrt - der litauische Reiter, rechts in einem weniger geläufigen Wappen Litauens die liegende Chiffre des Druckers $H V$, die sich über der unteren Randleise wiederholt ${ }^{185}$, vgl. dazu Abb. 44. Der Text ist komplett, die 1884 Begriffe des Dialogus sind - ähnlich den anderen gedruckten Überlieferungen - auf 1438 gekürzt.

Die Reihenfolge des Nomenklators ist bis zum Blatt $18^{r}$ des Trialogus völlig ident. Es folgt hier eine Gegenüberstellung der einzelnen Kapitel, wobei die Anordnung des Druckes mit fingierter Numerierung in eckiger Klammerung maßgebend ist. Dadurch erleichtert sich nämlich ein Vergleich mit der Arbeit von B. Wižd’álková, insbesondere mit ihren Synopsen zu fünf weiteren Drucküberlieferungen ${ }^{186}$. Der Blattzählung vor den Trialoguskapiteln entspricht eine von mir in runder Klammer ergänzte Seitenzählung im nicht paginierten Dictionarius:

\begin{tabular}{|c|c|c|c|c|}
\hline \multirow{2}{*}{\multicolumn{2}{|c|}{$\begin{array}{l}\text { Trialogus von } 1489 \\
1^{\mathrm{r}} \text { (keine Überschrift) }\end{array}$}} & \multicolumn{3}{|c|}{ Dictionarius von 1513} \\
\hline & & $<1>$ & (1) & De celo \\
\hline & 1" Z. 9 De Actibus & & (1) & (keine Überschrift) \\
\hline & $2^{\mathrm{r}}, \mathrm{Z} .13$ De ventibus (!) & $<2>$ & (1) & De aere \\
\hline & \multicolumn{4}{|l|}{$2^{v}$, Z. 22 Capitulum de Regionib(us) } \\
\hline & Provinciarum & $<3>$ & (2) & $\begin{array}{l}\text { De terra metallis \& } \\
\text { lapidibus }\end{array}$ \\
\hline & $3^{\text {v }}$ Z. 19 De Ecclesia Et Ip(s)ius attinentis & $<4>$ & (2) & $\begin{array}{l}\text { De ecclesia \& } \\
\text { pertine }(n) \text { tys eiusdem }\end{array}$ \\
\hline & \multicolumn{4}{|c|}{$\begin{array}{l}\text { Genaue Beschreibung bei Michael DenIs, Nachtrag zu seiner Buchdruckergeschicht Wiens. Wien 1793, } \\
\text { Nr. 759. Das ganze Buch ist bei Wiž̆́ÁLKová, Vokabulář (zit. A. 165) im Abschnitt } 1 \text { der Abbildungen }\end{array}$} \\
\hline & \multicolumn{4}{|c|}{$\begin{array}{l}\text { Vgl. dazu A. Nayer, Wiens Buchdrucker-Geschichte } 1482-1882 \text {. Hrsg. von den Buchdruckern Wiens.1 } \\
\text { (1482-1682). Wien 1883, } 32 \text { und } 151 \text { mit Abb. und KĘDELSKA (zit. A. 2) } 23 .\end{array}$} \\
\hline & \multicolumn{4}{|l|}{ Vgl. WižĎÁLKová, Vokabulář (zit. A. 165) 146-147. } \\
\hline
\end{tabular}


$4^{\mathrm{v}}, \mathrm{Z} .1$ De diebus

4v, Z. 22 De Papa et Alys Spiritualibus

$5^{\mathrm{v}}, \mathrm{Z} .4$ De doctoribus

$6^{\mathrm{r}}, \mathrm{Z} .11$ De carmi $(n)$ e et ip $(s)$ ius attinencys

$6^{\mathrm{v}}, \mathrm{Z} .2$ Capitulu(m) de $\operatorname{Imp}(\mathrm{er})$ io et

Regio(n)ib(us)

$7^{\mathrm{r}}$, Z. 11 De Judicio

8v, Z. 1 De Civib(us)

$8^{\text {v }, ~ Z . ~} 25$ De Ciuitate

$9^{\mathrm{v}}$, Z. 8 De mechanicis

$10^{\mathrm{r}}, \mathrm{Z} .21$ De armis

11 ${ }^{\mathrm{r}}, \mathrm{Z} .11$ De rustico \& alys p(er)tine $(n)$ cys

$11^{\text {v }, ~ Z . ~} 2$ De curro et alys attin(en)cys

12r, Z. 9 De Aratro et ip (s)ius p(er)ti(n)encys

13 ${ }^{\mathrm{r}}, \mathrm{Z} .6$ De frumentis

$13^{v}$, Z. 11 De herbis et ip (s)ius attinentis

$14^{\mathrm{r}}, \mathrm{Z} .22$ De vinea et alys pertinentys

14", Z. 13 De de(!) Arboribus

15 ${ }^{\mathrm{r}}, \mathrm{Z} .13$ De condicione Arboru (m)

16 ${ }^{\mathrm{r}}, \mathrm{Z} .6$ De Homi $(n)$ e $(m)$ et que ad ip $(s)$ um p(er)tinet

$18^{r}$, Z. 3 De Monstris

\begin{tabular}{|c|c|c|}
\hline \multirow{3}{*}{$\begin{array}{l}<5> \\
<6>\end{array}$} & (2) & De festis \& ferijs \\
\hline & (3) & De statu spirituali \\
\hline & (3) & (keine Überschrift) \\
\hline$<y>$ & (3) & De scriptoribus \\
\hline \multirow[t]{2}{*}{$<8>$} & (3) & De imperatoribus \\
\hline & & $p(r o) u i(n) \operatorname{ciar}(u m)$ \\
\hline \multirow[t]{3}{*}{$<9>$} & (4) & De iudicialibus \\
\hline & (4) & (keine Überschrift) \\
\hline & (5) & (keine Überschrift \\
\hline$<10>$ & (5) & De artificibus \\
\hline$<11>$ & (5) & De armis \\
\hline \multirow[t]{3}{*}{$<12>$} & (6) & De rusticis \& \\
\hline & & pertinentijs eorum \\
\hline & (6) & (keine Überschrift) \\
\hline$<13>$ & (6) & De agris \& suis \\
\hline & & pertine $(n)$ tijs \\
\hline $4>$ & $(\%)$ & De frumentis \\
\hline$<15>$ & $(y)$ & De herbis siluestrib(us) \\
\hline$<16>$ & $(y)$ & De vineis \& ortibus \\
\hline & (8) & (keine Überschrift) \\
\hline$y>$ & (8) & De arboribus \\
\hline & (8) & Sequitur de homine \\
\hline $9>$ & (9) & de monstris \\
\hline
\end{tabular}

Ab $18^{v}$ setzt der Druck mit einem Block fort, der im Trialogus erst später - ebenfalls als Block - folgt:

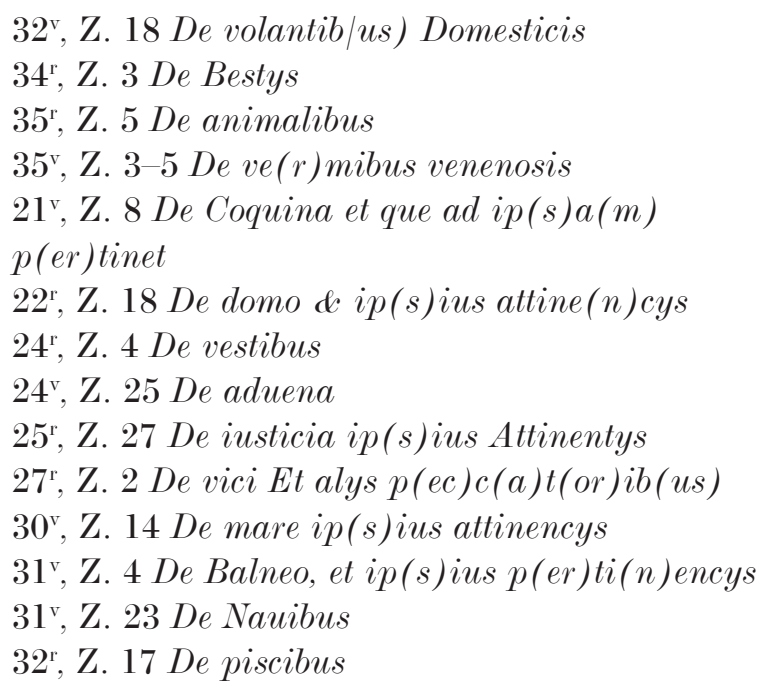

Die folgenden Kapitel laufen wieder parallel:

18v, Z. 1 De Genealogia et alys attine(n)cys 19r ${ }^{\mathrm{r}}$ Z. 20 De varys Infirmitatibus $20^{\mathrm{r}}$, Z. 7 De apothecis
$<20>$ (10) De auibus

$<21>$ (10) De quadrupedibus

$<22>$ (11) De bestiis \& feris

$<23>$ (11) De vermibus venenosis

$<24>$ (11) De coquina

$<25>$ (11) De domo

$<26>$ (12) De vestibus hominu $(m)$

$<2 \%>$ (13) De nominibus

$<28>$ (13) De virtutibus

$<29>$ (14) De vicijs

$<30>$ (15) De aquis \& mari

$<31>$ (15) De balneo \& pertinentys

$<32>$ (15) De nauibus

<33> (15) De Piscibus

$<34>$ (16) De Genealogia

$<35>$ (16) De Morbo

$<36>$ (16) De Apoteca 
Den Abschluß bilden im Druck:

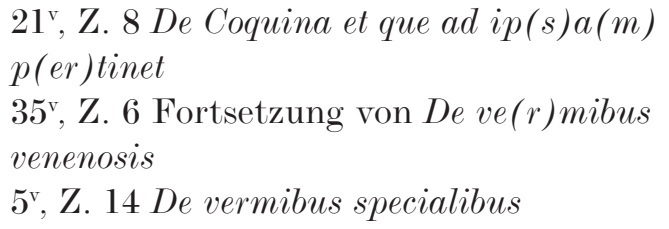

Im Vergleich zur vierundzwanzig Jahre älteren handschriftlichen Überlieferung ist nur das Kapitel De ve(r)mibus venenosis auseinandergerissen. Die blockartigen Differenzen in der Anordnung der Kapitel scheinen - wie gleich gezeigt werden wird - mit einem Lagenfehler in der bereits genannten Strahover Vorlage zusammenzuhängen ${ }^{187}$, die auffälligsten und typologisch wichtigsten Kürzungen bringt der Abschnitt de monstris ${ }^{188}$.

Bereits im Abschnitt über den Trialogus wurde erwähnt, daß im Druck von 1513 ungebräuchliche Verdeutschungen und Unklarheiten ausgemerzt oder gebessert, Dialektismen und Grobheiten „,verfeinert“ sind, vgl. etwa den Ersatz der bairischen Kennwörter oder Esula/scheyßwurtz (14, Z. 16) das im Druck zu wolffsmilch wurde Es wird eine reizvolle Aufgabe für künftige Forschungen sein, sprachliche Modernisierungen, Verallgemeinerungen oder Präzisierungen der jüngeren Tradition(en) systematisch festzumachen. Ich nenne hier nur Horalogium/worlog/zeyger statt Hur/Hodinne (4 ${ }^{\mathrm{r}}, \mathrm{Z}$. 25), worin sich Unsicherheiten angesichts des Wandels im Zeitverständnis im Zusammenhang mit der Erfindung der mechanischen Uhr spiegeln dürften ${ }^{189}$. Erwähnt seien ferner die tschechische Präzisierung Crabat für die alte Klaretgleichung Crabatus/Zrbeny $\left(25^{\mathrm{r}}, \mathrm{Z} .21\right)$, tschechisch žalarž statt Carcer/Schatlaw $\left(8^{v}, Z\right.$ Z. 22), vernufft statt racio/redlikait $\left(8^{r}, Z\right.$. 13) oder das „, hussitische“ hauffnitze statt Puska $\left(11^{\mathrm{r}}, \mathrm{Z} .7\right)^{190}$ usw. Weitere Beispiele haben Rudolf Wolkan, Václav Flajšhans und Bedřiška Wižd'álková gebracht ${ }^{191}$. In einigen Fällen ist aber der Trialogus auch genauer, vgl. etwa $15^{\mathrm{v}}$, Z. 22/23: Hier hat die Handschrift Boletus/Swam/pfiff(er)ling/Huba und Spongia/Padswam/ Muchomorz, wofür der Druck in beiden Fällen tschechisch nur hauba setzt.

Wenngleich man also vorsichtig geneigt ist, den Dictionarius trium linguarum als verbesserte Fassung des Trialogus zu bezeichnen, hat er - als Kind seiner Zeit und vermutlich Teil einer langen Überlieferungskette - doch jede Menge auch neuer Errata. Dies überrascht nicht, da auch in Wien die Pioniere der schwarzen Kunst gleichzeitig Drucker, Verleger und Buchhändler waren. In der ersten Zeit beschränkten sich die Buchhändler darauf, die Heilige Schrift oder vorliegende Manuscripte eines Donatus, eines Kirchenvaters, eines lateinischen Autors oder sonst beliebte Manuscriptbücher nachzudrucken, und zwar meist sclavisch, mit allen Abbreviaturen und Fehlern, wozu noch die der Druckerei selbst kamen ${ }^{192}$.

187 WižDÁlkowa, Vokabular (zit. A. 165), 23-52.

188 Ebenda, 22

189 Eine Tullner Urkunde von 1372 verbalhornt horologium zu deutsch arloy (!) und meint eine mechanische Schlaguhr am Turm der Stadtpfarrkirche, vgl. ENGLisch - JARITz, Das tägliche Leben (zit. A. 87) $12-13$.

190 Vgl. dazu Bellmann, Slavoteutonica (zit. A. 85). 276-277; Vintr, Das Tschechische (zit. A. 13), 157; E. Wagner - Z. Drobná - J. Durdík, Tracht, Wehr und Waffen des späten Mittelalters (1350-1450) Prag $1957,62$.

191 S. Wolkan, Geschichte der deutschen Literatur (zit. A. 91) 92-93; V. FlaJŠHans, Klaret (zit. A. 75) XXXII; WiŽĎÁLKová, Vokabulař (zit. A. 165) 135-136.

192 MaYer, Buchdrucker-Geschichte (zit. A. 184) 155. 
Tatsächlich gibt es eine große Zahl von Eigenfehlern, vgl. etwa vndertenigkeit gegen Qualitas/Wietennkhait der Handschrift (2r, Z. 3), pester statt pesler bzw. Pasler (10 $\left.{ }^{\mathrm{v}}, \mathrm{Z} .26\right)$, wohl auch Verhenngnüs statt Gehengnüs $\left(28^{\mathrm{r}}, \mathrm{Z} .6\right)$, die Fehlübersetzung hubscheyt zu Facundia/ wymluwuost, wo die Handschrift Beredigkait/Snaznost hat (26r, Z. 17) u. a. Ein Kabinettstück ist schließlich 22r, Z. 16, wo der Kopist zunächst lat. Licinum mit Limen verwechselt. Der Trialogus schreibt Licinu $(m) /$ Tacht/Knot, im Dictionarius steht Limen/prah/zacht, statt zu erwartendem deutschem schwelle wurde eine nicht verstandene Entsprechung für „Docht“ einfach als zacht gesetzt. Beweisend für diese schluderhafte Zeilenverkürzung ist die diesmal richtige Wiederkehr der Limengleichung wenige Zeilen später auf $22^{v}, 2^{193}$.

Am augenfälligsten unter vielen Druckfehlern sind aber die zahlreichen u-n bzw. w-mn-Vertauschungen, die übrigens den Buch- und speziell den Glossardruck von Anfang an begleitet haben ${ }^{194}$, vgl. kadidluitze (4 ${ }^{\mathrm{r}}, \mathrm{Z}$. 18, Trialogus Kadelnicz), žuetz (11 ${ }^{\mathrm{r}}, \mathrm{Z} .22$, Snecz),

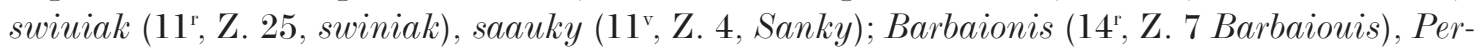
sicus břeskew (15 $5^{\mathrm{r}}$ Z. 4, Brzesken), Ulua (16 ${ }^{\mathrm{r}}$, Z. 1,Vlna), schmeißtuch $\left(24^{\mathrm{r}}\right.$, Z. 8, Swaistuch) u.a. Verbreitet sind auch r-t-Verwechslungen, etwa počarek (1 ${ }^{\mathrm{v}}, \mathrm{Z} .14$, Poczatek), žalrarz $\left(4^{\mathrm{r}}, \mathrm{Z} .20\right.$, Saltarz), Bartha (32, Z. 12, Barca), vgl. auch Cateburca $\left(10^{\mathrm{v}}\right.$, Z. 18 Cataburta). Nicht selten sind ferner Verwechslungen des langen s mit f, z. B. in Sopitus-verschlossen ( $7^{\mathrm{v}}$, Z. 2, Verslaffnn), przeflitze (23v, Z. 27, Przieslicze), prafatko (34' nach 24, tschech. prasátko), Coluber-flepetz $(35$, Z. 8, zu tschech. slep, im Trialogus Schewad). Daneben gibt es jede Menge anderer Mißverständnisse, vgl. Tegula (23v , Z. 1, Regula), Petriscus (34', Z. 1, Pistricus), birtken (15' , Z. 14, Pirkhnn), Monocul(us)/einau statt einaug (nach 18 ${ }^{r}$, Z. 14), staha $\left(24^{r}, Z\right.$ Z. 13, Wztuha), reysky (25', Z. 24, Reynsky) usw.

Für den tschechischen Teil ist ein Überhandnehmen der digraphischen Schreibungen für die Diphthonge ou, uo aus ú, ó ersichtlich, vgl. etwa korauhew (3 ${ }^{\mathrm{v}}, \mathrm{Z} .27$, die Handschrift hat Koruheb), chlauba $\left(27^{\mathrm{r}}\right.$, Z. 19, Chluba) pausciadlo (31 ${ }^{\mathrm{v}}, \mathrm{Z} .10$, Pusczadlo), auhorz (32', Z. 12, Vhorz), kohaut (32', Z. 18, Kohut), dwuor (12v' Z. 2, Dwor), ruože (13', Z. 13, Rozie), kuora (14", Z. 6, Kora) usw. ${ }^{195}$, wobei gelegentlich auch ein deutsches Lemma „mitgenommen“ wird, vgl. smetana/milchraum (34v nach 14). Vokaldehnungen werden häufiger als im Deutschen durch Gemination angedeutet, z. B. hraach, yaahly, maak (13', Z. 10/12) oder weselee (25' ${ }^{\mathrm{v}}$ Z. 8) u.a., vgl. dazu deutsch keel (16 ${ }^{v}$, Z. 26). Allerdings kennt der Drucker auch bereits Akzentzeichen, vgl. etwa missál (nach 4r, Z. 19), kázeň (6r, Z. 8), hrácz/spiler $\left(29^{\mathrm{v}}\right.$, Z. 13) u.a. bzw. deutsch Miluus/áher (33'r, nach19), wáden (17', Z. 18).

Der wohl sichtbarste Unterschied gegenüber der Handschrift aber ist das Vordringen der diakritischen Orthographie und insbesondere des Hatscheks zur Festlegung der Palatalisierung, was wohl auch mit den Normierungstendenzen des neuen Druckmediums zu tun hat,

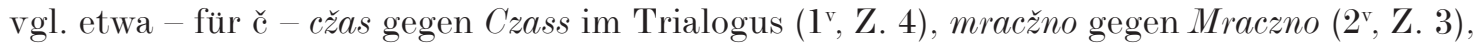
porucženi gegen Poruczeny ( $\left.7^{\mathrm{r}}, \mathrm{Z} .19\right)$ usw. - für ĕ slepitzĕ gegen Slepitze (32v, Z. 19), für ň kázeñ gegen Kazan (6 $6^{r}, Z$. 8), kuon̆ gegen Kun $\left(34^{r}, Z\right.$ Z. 11) - für ř - stwořeny gegen Ztworzenye (1 ${ }^{v}, Z$ Z. 14), kopřywa gegen Koprziwa $\left(13^{v}\right.$, Z. 15), třessnie gegen Trziessnye (14v, Z. 25) bzw.

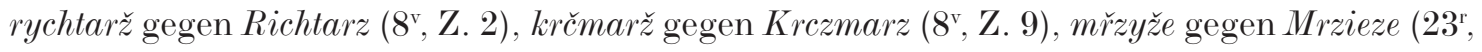

193 Vgl. dazu WižĎálková, Vokabulář (zit. A. 165) 58.

194 Vgl. Introito e Porta compiuto per Meistro Adamo de Roduila, 14 \%' adi 12 Augusto. Prefazione di A. BarT Rossebastiano (Neolatina rariora I/Lexicalia 1) Torino 1971 (Nachdr.) 21 Dieuen/Seruir u.a.; Lexikon symphonon Sig. Gelenii iam duplo auctius. Basel 1544, 1 agmen/hauf usw.

195 Dazu Wižð̌álková, Vokabulař (zit. A. 165) 93-94; allgemein Vintr, Tschechisch (zit. A. 13 ) 153. 
Z. 17) usw. - für ž kniže gegen Knyze (6 ${ }^{v}, Z$ Z. 13), lžytze gegen Lssycze $\left(20^{\mathrm{v}}, \mathrm{Z} .22\right)$, žoldowani gegen Zold $\left(9^{r}\right.$, Z. 27). Somit ist das Buch des Viëtorius - wohl in Nachfolge des Strahover Bruchstücks - ein frühes Druckbeispiel des Weges zum Schreibusus der „Böhmischen Brüder"196. Es ist übrigens zumindest erwähnenswert, daß der deutsche Text die sonst üblichen Diakritika vermeidet, auf $34^{\mathrm{r}} \mathrm{Z}$. nach 2 als kurioser Satzfehler aber deutsch galandeř für lateinisch Fenix steht.

Die Wiener Auflage des dreisprachigen Vokabulars von 1513 entsprang einer geschäftlichen Kalkulation und mußte sich verkaufen. Dabei dürfte die zugrundeliegende Vorlage kaum nur eines der vorhin zitierten und in Wien „beliebten Manuscriptbücher" gewesen sein, etwa eine gängige lateinisch-tschechisch-deutsche Wortliste, wie sie in ähnlicher Form schon 1489 für Kaiser Maximilian kopiert worden war. Aufgrund des vermutlich vom Strahover Vokabularfragment übernommenen und auch 1514 im Leipziger Vocabularius hic cuique perutilis nachgedruckten Formfehlers - ${ }^{197}$ steht ja fest, daß der Dictionarius - anders als früher vermutet - keineswegs für sich steht ${ }^{198}$.

Diese endgültige Klärung gelang B. Wižd’álková nach einer Autopsie in der Strahover Prämonstratenserbibliothek. Das dortige Bruchstück 554/zl (vgl. Abb. 42) besteht aus zwei Resten eines Terniobogens, dessen Fehlerhaftigkeit durch falsches Ansetzen des Drucksatzes auf die Rückseite noch zu erkennen ist. Trotz starken Beschnitts und obwohl das mittlere Doppelblatt vollkommen fehlt, läßt sich feststellen, daß es sich um Rudimente eines lateinisch-tschechisch-deutschen Glossars handelt, die mit Viëtors Dictionarius trium linguarum von 1513 nahezu ident sind. Beide Vokabulare teilen gegen den Trialogus alle Auslassungen, Interpolationen, tschechischen Diakritika und Überschriften, aber auch ganz augenfällige grobe Fehler, z. B. lateinisch Nidus statt Nisus (33' ${ }^{r}$ Z. 21), tschechisch gywokimuož statt divoký muž (18, Z. nach 4), deutsch einau statt einaug (18, Z. nach 14) oder den Irrläufer Cumes/husze/wecke „(Weiß)brotwecken“ (35" , Z. nach 5) im Kapitel de vermibus venenosis, übrigens mit Verbalhornung des lateinischen Cuneus. Viëtor hat gegenüber dem Strahover Text nur in einem Fall (Strahov, Folium $1^{\mathrm{b}}$ bzw. vermutlich $5^{\mathrm{a}}$ ) einige Zeilen en bloc ausgetauscht. Natürlich gibt es punktuelle Differenzen in allen drei Sprachen, etwa lateinisch Jus des Strahover Bruchstücks gegen Prodium im Wiener Druck (21 $\left.{ }^{\mathrm{r}} \mathrm{Z} .17\right)$, tschechisch rod statt plod $\left(19^{\mathrm{r}}, \mathrm{Z} .18\right)$ oder deutsch gerucht statt geschmack $\left(20^{\mathrm{r}}, \mathrm{Z} .12\right)$ etc., dazu die üblichen individuellen Flüchtigkeitsfehler, vgl. Antipites statt Antipodes (18 ${ }^{\mathrm{r}}$, Z. 13), ktoznicznaumidielati statt ktoznitzneumidielati $\left(18^{\mathrm{r}}, \mathrm{Z}\right.$. nach 4 , tschechisch ktožnie neumi dělati, „der nicht machen kann“), deutsch schwaum statt schaum (31', Z. nach 6) etc.

Das Strahover Fragment enthält nur die fragmentierten äußeren Doppelblätter des Dreifachbogens, also Folium $1-2$ und 5-6, während der innere Teil mit Blatt $3-4$ fehlt, doch läßt sich daraus ein Umfang von $17^{\mathrm{r}}$, Z. 7 bis zum Schluß des Buches auf $36^{\mathrm{r}} 11$ erschlie-

196 Vgl. J. Schöpfer, Hussens Traktat „Orthographia Bohemica“. Die Herkunft des diakritischen Systems in der Schreibung slavischer Sprachen und die älteste zusammenhängende Beschreibung slavischer Laute (Slavistische Studienbücher IV). Wiesbaden 1968, 27-29; VINTR, Tschechisch (zit. A. 13) 160. S. dazu auch die Liste zum Schreibusus im Trialogus bei F. Menčík, Prešpurský slovník (zit. A. 162) XXVI-XXVII.

197 WižĎÁcková, Vokabulař (zit. A. 165) besonders 45-53 mit erklärenden Skizzen.

198 Vgl. allerdings schon H. Lopaciśski, Najdwniejscze slowniki polskie drukowane. Prace filologiczne V (1897) 5. B. Ryвa, der Entdecker des Strahover Fragments, erkannte die Zusammenhänge mit dem Wiener Druck von 1513, nicht aber mit dem Trialogus, vgl. seine leider ungedruckte Abhandlung Quelques mots sur le fragment no 554/\%l. Prag 1968. Das Typoskript liegt dem Fragment bei. 
Ben. Der Druckbogen enthielt damit annähernd die Hälfte des Trialogustextes bzw. des Dictionarius ohne Numerus ${ }^{199}$ Das erlaubt die Vermutung, daß davor ein erster Ternio existiert haben könnte, der verlorenging. Es scheint somit eine komplette Vorlage für den Dialogus von 1513 gegeben zu haben, die - nach dem Fundort zu schließen - durchaus in Prag entstanden sein könnte. In diese Richtung mag übrigens die ostmitteldeutsche Schreibung geheiltferd statt geheiltpferd (34, Z. nach 12, vgl. auch S. 55) weisen. Weitere Eingrenzungen sind derzeit leider nicht möglich, es ist kein Wasserzeichen erkennbar und die Lettern differieren von allen zeitnahen Vokabulardrucken, die eingesehen werden konnten. Sollte sich die vorsichtige Zuschreibung aber einmal bestätigen, so wäre eine lange Prager Glossartradition nach Klaret erwiesen, möglicherweise auch ein pragerdeutsches Sprachdenkmal in der dunklen Zeit nach den Hussitenwirren. Die kärgliche Quellenlage aber darf nicht verwundern, denn Sprachbehelfe der vorliegenden Art waren Gebrauchsgegenstände und entsprechendem Verschleiß ausgesetzt. In jedem Fall aber ist das Bruchstück von größtem Wert für die Bohemistik, und es steht zu hoffen, daß es ehestens restauriert und auch ediert werden kann.

Bedřiška Wižd'álková hat überdies - bis zurück in die neunziger Jahre des fünfzehnten Jahrhunderts - noch weitere Vokabulare verglichen, die in der Trialogustradition stehen müssen, denen allerdings der tschechische Text fehlt. Genauer untersucht wurden die Vocabula pro iuvenibus, gedruckt in Augsburg um etwa 1505 bzw. in Nürnberg 1508, und Vocabula rerum variarum aus Leipzig um $1495^{200}$. Sie haben gemeinsame sprachlich-formale Eigenheiten, stehen vor allem in der Binnengliederung dem Trialogus am nächsten und lassen auf weitere Zwischenglieder schließen. Bezüglich der Genesis von Viëtors Druck von 1513 kann mit Sicherheit gesagt werden, daß er nicht durch eine direkte Bearbeitung des Trialogus entstanden ist. Daß indes ,... die dreisprachigen Vokabulare aus den Vocabula durch Hinzufügung tschechischer Termini entstanden (sind) ..." ist, schon wegen der Vollständigkeit des tschechischen Abschnittes und seines ausschließlichen Klaretbezugs für den Trialogus weniger wahrscheinlich ${ }^{201}$.

Im Anschluß sei versucht, derzeit bekannte Inkunabelausgaben der Vocabula pro iuvenibus zusammenzufassen: Es gibt acht verschiedene Leipziger Drucke, darunter die vermutlich ältesten: Sie stammen von Conrad Kachelofen (zwischen 1489 und 1497, zwei Auflagen) bzw. Gregorius Böttiger (um 1495, zwei Varianten), Melchior Lotter (1499 bzw. 1500) und Landsberg (undatiert). Weitere sechs Wiegendrucke stammen aus Augsburg, Nürnberg und Basel ${ }^{202}$. Die Distribution der lateinisch-deutschen Drucke zeigt also Leipzig als Zentrum des Vocabula pro iuvenibus-Typs, was angesichts historischer Zusammenhänge zwischen Böhmen und Sachsen nicht überrascht. Beispielsweise druckte Conrad Kachelofen schon 1483 eine Passio Pragensium, 1498 das Missale Pragense. Die offensichtliche Abhängigkeit der schulisch orientierten

199 Ebenda, 100-131, eine Zusammenstellung von Texdifferenzen zwischen dem Strahover Bruchstück und den nachfolgenden Drucken von 1513, 1514 und 1532.

200 Vgl. ebenda, 18-19 bzw. Abb.3-5, ferner F. ClaEs, Bibliographisches Verzeichnis der deutschen Vokabulare und Wörterbücher, gedruckt bis 1600. Hildesheim-New York 1977, Nr. 114.

201 Wiž̄̌álková, Vokabulař (zit. A. 165) 158. Vgl. auch 20-22 und passim.

202 Vgl. dazu unser Literaturverzeichnis, zusammengestellt nach Wiž̌̌ÁLKová, Vokabulař (zit. A. 165) 139, F. Clates, Verzeichnis (zit. A. 200) und dem ISTC der British Library in London, dem weltweit recherchierten EDV-Katalog der Frühdrucke. Strittige Datierungen folgen dem ISTC. Neue Ergebnisse für das 16. Jahrhundert bei P. O. Mứler, Deutsche Lexikographie des 16. Jahrhunderts. Konzeptionen und Funktionen frühneuzeitlicher Wörterbücher (Texte und Textgeschichte 49) Tübingen 2001, 305 und 308 , Fn. 25 
Vocabula pro iuvenibus mit dem für die Karlsuniversität erstellten Claretus bzw. seinem Auszug im Trialogus läßt sehr vorsichtig weiterdenken: Es mag sogar ein Zusammenhang mit der Verlegung der Prager (deutschen) Universität nach Leipzig in der Folge der Kuttenberger Dekrete von 1409 bestehen. Damit könnte man die spezifischen Gemeinsamkeiten der Vocabula und insbesondere die nur mehr lateinisch-deutsche Zusammenstellung interpretieren. Man müßte allerdings annehmen, daß es damals bereits einen im Verhältnis zur Trialogushandschrift etwas kürzeren Wortextrakt als Ausgangspunkt aller bekannten Drucke gab ${ }^{203}$. Ein autochthoner Prager Zweig mit den Ergänzungen des Trialogus könnte - als Vorlage auch für das Strahover Fragment, (bei dem das Tschechische vor dem Deutschen steht), bis an die Donau gewirkt haben.

Die Wiener Ausgabe von 1513 ist - wie bereits erwähnt - der früheste komplette Glossardruck, der das Tschechische zum Lateinischen und Deutschen einbezieht. Treibende Kraft hinter der Vervielfältigung war zweifellos Hieronymus Viëtor (Hieronim Wietor), wie sein schon erwähntes großes Druckzeichen am Schluß des Buches anzeigt ${ }^{204}$. Viëtor, auch Philovallis und Doliarius, mit deutschem Namen Büttner, stammte aus Liebenthal im schlesischen Fürstentum Jauer und studierte in Krakau, wo er 1499 das Baccalaureat machte. Gleichzeitig erlernte er den Buchdruck und knüpfte damals vermutlich seine engen Beziehungen zur Stadt und zum polnischen Adel. 1510 eröffnete er einen Buchladen und begann für Krakaus Studenten Lehrbücher zu drucken. Fast gleichzeitig gründete er mit Johann Singriener in Wien eine Offizin. Dieses Unternehmen der laborum et lucri socii hielt bis Ende 1514 ${ }^{205} .1517$ kehrte Viëtor nach Krakau zurück, vielleicht wurde ihm der Wechsel durch die Empfehlung polnischer Aristokraten erleichtert, die mit König Sigismund 1515 zur Wiener Doppelhochzeit gekommen waren ${ }^{206}$. Neben der neuen Offizin in Krakau, wo er 1541 starb, hielt Viëtor aber auch die Wiener Druckerei bis 1531 aufrecht.

Viëtors Interesse an einem Kommunikationsbehelf unserer Art kann nicht überraschen: Als nüchterner Geschäftsmann, zugleich aber gelehrter Kenner des slawisch-deutschen Interferenzbereichs erfaßte er den Bedarf eines dreisprachigen Drucks mit dem Tschechischen, das damals auch im östlichen Mitteleuropa die führende slawische Hochsprache war ${ }^{207}$. Leider läßt sich Michael Denis Vermutung, die Ausgabe sei durch die 1513 schon angelaufenen kaiserlichen Unterhandlungen mit Wladislaw II. veranlaßt worden, nicht überprüfen ${ }^{208}$. Fest steht nur, daß das Buch peregrinantibus apprime vtilis sein sollte und daß Vietors Biographie und Lebenswerk durchgehend eine Neigung zum slawischen Kulturbereich erkennen lassen. Das zeigt schon sein erster Verlagsauftrag an Johann Winterburger in Wien, bei dem 1509 die Gedichte des Ruthenen Paul von Crosna (Krosnyanin) an Sankt Ladislaus von Ungarn bzw. Sankt Stanislaus von Polen herauskommen. Sein Briefdruck von 1511, der mit Roderici

203 Vgl: die Tabelle bei WižĎálková, Vokabulař (zit. A. 165) 33-34 bzw. 100-130.

204 Auch zum Folgenden vgl. Mayer, Buchdrucker-Geschichte (zit. A. 184) 152 und 162

205 In diesem Konnex belanglos ist der Umstand, daß spätere Auflagen unter dem Namen Singrieners erschienen. Singriener und Vïetor druckten einander nach der geschäftlichen Trennung verschiedentlich nach, ein Zeichen, daß sie Freunde blieben. Vgl. M. Denis, Wiens Buchdruckergeschicht bis MDLX. Wien 1782, VIII. Zur Biographie vgl. auch J. PTaśnik, Cracovia impressorum XV et XVI saeculorum (Monumenta Poloniae typographica XV et XVI seculorum I) Lemberg 1922, 37-42.

206 S. M. Denis, Wiens Buchdruckergeschicht bis MDLX. Wien 1782, VIII.

207 Dazu Vintr, Das Tschechische (zit. A. 13) 158-159, 165.

208 Die genaueste Beschreibung dieses Ereignisses bei H. Wiesflecker, Kaiser Maximilian I. Das Reich, Österreich und Europa an der Wende zur Neuzeit 4. Wien 1981, 181 ff. 
Dubravi Bohemi Libellus de componendis Epistolis beginnt ${ }^{209}$ belegt dann die ganze Breite dieser Interessen. In seiner späteren Zeit hat sich Viëtor - mit Sicherheit auch kompilatorisch - mit polnisch-deutschen Gesprächsbüchern befaßt, übrigens in Zusammenarbeit mit einem schlesischen Landsmann, dem Humanisten Franciscus Mymerus (Franz Mymer). Jedenfalls ist es nicht zuletzt Viëtor zu verdanken, daß Krakau zum polnischen Zentrum für sprachvergleichende Drucke im sechzehnten Jahrhundert wurde ${ }^{210}$. Die polnische Literatur bekam durch ihn die ersten gedruckten Bücher in der Landessprache und orthographische Neuerungen, die durchaus in Parallele zum spřežkový pravopis unseres Trialogus gesehen werden können ${ }^{211}$.

Die Strategie Viëtors erwies sich als richtig, denn der Typ des Dictionarius trium linguarum war ein „Trendsetter" vielsprachiger Lexikographie mit slawischer bzw. auch ungarischer Beteiligung im sechzehnten Jahrhundert. Ein Nachdruck des Dictionarius kam bereits ein Jahr nach der Wiener Auflage in Leipzig heraus, Peter O. Müller konnte die undatierte Ausgabe Vocabularz Latine e Cescy a Niemetzky kaz dému mlademu y Staremu welmi potrzebny a vzyteeny. Vocabularius rerum aus der Nürnberger Offizin Jodocus Gutknechts nachweisen, und noch 1532 erschien ein Druck bei Johann Peck in Pilsen. Viëtor hat damit auch direkt auf die polnische Lexikographie eingewirkt ${ }^{212}$. Eine unmittelbare Übernahme des Dictionarius durch vier- bis sechssprachige Publikationen dieser Art, wie seit Josef Jungmann und noch von Franz Claes in der tschechischen, deutschen und auch ungarischen Literatur immer behauptet wurde, läßt sich indes nicht erkennen, womit auch Spekulationen über einen Zusammenhang mit der Tradition des venezianischen Introito e Porta Adams von Rottweil in sich zusammenfallen ${ }^{213}$.

Erst der 1531 in Nürnberg gedruckte Quinque Linguarum Vtilissimus Vocabularius, in dem einer italienischen bzw. französischen eine tschechische Kolumne hinzugefügt wurde, steht eindeutig in dieser von Venedig ausgehenden Überlieferung ${ }^{214}$, gleiches gilt für die 1538 wieder bei Singriener herausgekommene Nomenclatura sex linguarum, Latinae, Italicae, Gallicae, Bohemicae, Hungaricae \& Germanicae ${ }^{215}$, einem Pionierwerk der ungarischen Schriftsprache. Nebstbei könnte sie in Zusammenhang mit einschlägigen Arbeiten des Silvester Joannes Pannonius in der Krakauer Offizin Viëtors stehen ${ }^{216}$. Von dieser sechssprachigen Nomenclatura wird im nächsten Kapitel noch die Rede sein.

209 Denis, Wiens Buchdruckergeschicht (zit. A. 206) Nr. 52.

210 Dazu Kędelska, Slowniki (zit. A. 2) 107; P. O. Múller, Lexikographie (zit. A. 202) 308-310.

211 Dazu KęDelska, Slowniki (zit. A. 2) besonders 58 und 67-74; WižĎÁlková, Vokabulař (zit. A. 165) 140-145; MAYer, Buchdrucker-Geschichte (zit. A. 174) 30-37, mit einiger Vorsicht auch M. Bieder, Das polnisch-deutsche Vokabularium aus der Krakauer Offizin des H. Viëtor, gedruckt im Jahre 1539. Wien Diss. 1975, besonders 46 und 51 .

212 P. O. Mứlter, Lexikographie (zit. A. 202) 308, Fn. 25; KęDELsKa, Slowniki (zit. A.2) 59-60.

213 Vgl. ebenda 20, ferner A. Rossebastiano, Antichi vocabolari plurilingui d'uso popolare: la tradizione del „Solenissimo Vochabuolista“. Alessandria 1984, 256.

214 Dazu ebenda, Nr. XXVIII, bzw. die Erörterungen des Typs C, 239-246. Vgl. auch H. Brauner, Die tschechische Lexikographie des 16. Jahrhunderts. Diss. Breslau 1939, 31-39. Eine unwissentliche Bestätigung der Abhängigkeit gibt KĘDELska, Slowniki (zit. A. 2) 27, indem sie auf die völlige Abhängigkeit vom gleichnamigen venezianischen Druck Francesco Garonnes, Venedig 1526, aufmerksam macht, vgl. S.27-28 bzw. Abb. 6-13.

215 Rossebastiano, Antichi vocabolari (zit. A. 203) Nr.XXXVIII. Vgl. Mayer, Buchdrucker-Geschichte (zit. A. 174) 65 .

216 Vgl. KęDELSKA, Slowniki (zit. A. 2) 38-39. 
Der Druck Hieronymus Viëtors von 1513 steht zumindest mittelbar in der Überlieferungskette des Trialogus, geht also wie dieser auf einen Auszug aus dem Glossarius des Claretus zurück, dessen weitere Ausformungen noch $\mathrm{zu}$ erforschen sind ${ }^{217}$. In Konnex mit der Pappa puerorum des Münsteraner Pädagogen Johannes Murmellius, die über den Ostseeraum bis Skandinavien wirkte ${ }^{218}$, könnte man mit einiger Berechtigung sogar von nördlichen und nordöstlichen Gegentraditionen zum Introito Adams von Rottweil und seinen Filiationen sprechen.

Insgesamt aber wird ein Leitgedanke von Václav Flajšhans wieder verstärkt: Ausgangspunkt der nachfolgenden lexikalischen Arbeiten mit Beteiligung der tschechischer Sprache ist die große karolinische Versenzyklopädie des Claretus, ein Aspekt, den die Germanistik noch nicht übernommen hat. Diese Tradition lebt breitenwirksam im Dictionarius trium linguarum von 1513 weiter, dem mit Abstand einflußreichsten Sachgruppenwörterbuch des 16. Jahrhunderts. Der Einfluß auf die polnische Glossographie ist aber weniger geradlinig gelaufen, als lange Zeit angenommen ${ }^{219}$. Der enge Zusammenhang zwischen dem bairischösterreichischen Trialogus von 1489 und dem Wiener Dictionarius läßt zumindest nicht ausschließen, daß eine zugrundeliegende Tradition auch in der zeitweiligen Residenzstadt an der Donau verankert war.

217 Vgl. Wiž̌̌ÁLKová, Vokabulař (zit. A. 165) 138-140. Zu weiteren Krakauer Ausgaben Viëtors s. jetzt P. O. Mứlter, Lexikographie (zit. A. 202) 308-309

218 S. D. Reichling, Johannes Murmellius. Sein Leben und seine Werke. Freiburg/B. 1889, 93/94, Bibliographie 150-152. Vgl. ferner Variorum rerum vocabula cum sueca interpretatione. Formulae puerilium colloquiorum. Stockholm 1579. Faksimile... med en efterskrift av Isak CoLLIJN (Faksimileupplagor av äldre svenska tryck. VI) Stockholm 1925, 7-8; P. O. MüLlen, Lexikographie (zit. A. 202) 313-317

219 S. Fla.šHans, Klaret (zit. A. 75), besonders XXXII. Zur differenzierten Ausstrahlung auf die polnische Glossographie vgl. jetzt KęDELsKa, Slowniki (zit. A. 2), 59-74, zusammenfassend 106-108. Den Stand der Germanistik referiert P. O. Müller, Lexikographie (zit. A. 202) 306 